\title{
Cultural Competence Nursing Care and Its Associated Factors Among Nurses in Northern Ethiopia: A Mixed Method Study Design
}

\author{
Kumlachew Mulatie Berie \\ Mohammed Hassen Salih (iD \\ Hailemichael Kindie Abate \\ Department of Medical Nursing, College \\ of Medicine and Health Sciences, \\ University of Gondar, Gondar, Amhara \\ Regional State, Ethiopia
}

Background: Cultural competence comprises understanding the values, beliefs, traditions, and customs of diverse groups. Nurses working with a culturally diverse population are fronting challenges principally due to the coexistence of multicultural and multiethnic societies.

Objective: To assess cultural competency nursing care and its associated factors among nurses working in Amhara Regional State Referral Hospitals, Ethiopia, 2021.

Methods: Institution-based mixed method study design was conducted at selected referral hospitals in Amhara Regional state Ethiopia from April 1 to May 30, 2021. A simple random sampling technique was used to select 543 participants for quantitative and purposive sampling for qualitative study. Data were collected using a self-administered Nurse Cultural Competence Scale Questionnaire, and semi-structured questions for in-depth interviews. Data were entered into Epi data version 4.6 and exported to SPSS version 25 analysis. Descriptive statistics independent $t$-test and one-way analysis of variance with multiple linear regression analysis were used for data analysis. Qualitative data were analyzed using thematic analysis using open code software.

Results: A total of 543 participants were involved in this study making a response rate of $95.02 \%$ and seven participants for qualitative until data saturation was involved. More than half (54.5\%) were female. The overall cultural competence nursing care of mean score was 3.39 ( 0.61$)$ out of a total possible score ranging from 1 to 5 which indicates a moderate level of cultural competency. The language barriers, lack of organizational support, cultural difference, and health care provider-related factors were the main identified themes in qualitative results.

Conclusion and Recommendation: The overall cultural competence was at a moderate level, among four subscales cultural sensitivity was the highest. The language barrier and lack of training were the major barriers to culturally competent nursing care.

Keywords: culture, cultural competence, nursing care, cultural diversity, Ethiopia

\section{Introduction}

Cultural competence is an ongoing process in which the health care provider continuously attempts to achieve the ability to effectively work within the cultural context of an individual, family, or community from a diverse cultural background. ${ }^{1}$ It is attributed to cultural awareness, cultural sensitivity, cultural knowledge, and cultural skill. ${ }^{2,3}$

Globally, the major cause of health disparities is the existence of cultural diversity. Studies showed that providing culturally competent care is an influential tool to eliminate the long-standing disparities. ${ }^{4}$ Twenty million nurses across the globe and four million nurses in the United States provide high-quality,

\footnotetext{
Correspondence: Hailemichael Kindie Abate

Department of Medical Nursing, College of Medicine and Health Sciences, University of Gondar, PO Box 196, Gondar, Amhara Regional State, Ethiopia Tel +251965321790

Email haile206k@gmail.com
} 
transformative health care to an ever more diverse population. ${ }^{5}$ A systematic review conducted in Canada, New Zealand, Australia, and the United States showed that $31 \%$ of the participants had poor culturally competent nursing care. ${ }^{6}$ A cross-sectional study conducted on Sudanese nurses revealed that about $58.7 \%$ of the participants had low cultural competency scores. ${ }^{7}$ A study conducted in Ethiopia among 270 nurses showed that only $57.3 \%$ had good cultural competency nursing care. ${ }^{8}$

Health-related errors such as unintended act (omission of plans, miscommunication), or one that does not achieve its intended outcome, failure to plan action to be completed as intended, use of wrong plan to achieve the goal, or deviation from the action of the care that may harm care the patients. These health-related errors were the third leading cause of death in the United States and had a magnitude of more than 250,000 deaths each year. Communication errors related to language and cultural differences are a major cause of these mistakes. ${ }^{9}$ Cultural competence is viewed as a critical factor and essential component in providing relevant, effective, and culturally responsive healthcare services to a diverse population. ${ }^{10}$ Patients from numerous cultural circumstances with diverse health-related characteristics, traditions, healthcare requirements, and expectations become increased in all the nations. ${ }^{3}$ The investigation now a day confirms that training is an actual way of cultivating providers' awareness of cultural and behavioral aspects of health care. The impact that health care providers who lack cultural competence may be putting patients at risk for delaying in treatment, inappropriate diagnoses, noncompliance with health care regimens, and even death. ${ }^{11}$ Patients with diverse cultures and languages get access to health care services in a less manner than the host population and are provoked for different barriers such as communication barriers. To decrease these inequalities, the promotion of cultural competence in healthcare organizations has been a political objective for several nations. $^{12}$

Growing diversity makes opportunities and challenges for healthcare experts, healthcare services and health policies to develop and carry out culturally competent care. ${ }^{13}$ Evidence revealed that the presence of cultural difference was found as a factor that negatively affects the nursing intervention. In some cases, clients refused intervention due to cultural differences. An insufficiency of knowledge of clients' language abilities and cultural views and beliefs can result in serious threats to life and less quality of care. ${ }^{14,15}$ The standards for culturally competent nursing care recommend that health care organizations should provide the structure and resources necessary to assess and meet the cultural and language needs of diverse clients in nursing care. ${ }^{16}$ In Finland, the necessity for teaching cultural competence in nursing education has been recognized and guidelines for recognizing cultural content in the nursing curricula have been ended to solve such difficulties. $^{17}$

In Ethiopia, there is a culturally diverse population, however, up to the knowledge of researches, there were limited studies as well no study conducted in the study area to show the magnitude of the problem that nurses face during giving care to such a culturally diverse population. Therefore, this study aimed to assess the magnitude of cultural competence nursing care and to explore the factors of culturally competent nursing care.

\section{Methods}

\section{Study Design, Study Setting, and Sample}

An institution-based cross-sectional quantitative study triangulated with qualitative approach (mixed method) was conducted. The study was conducted from April 1 to May 30, 2021.

The study was conducted among four randomly selected referral hospitals in Amhara Regional State, Ethiopia. Each referral hospital serves around 3.5-5 million people. The total number of nurses working in these selected referral hospitals were 1372 .

The source population for this study was all nurses who worked at the referral hospitals in Amhara Regional State, whereas the study population was all nurses who worked at selected referral hospitals in Amhara Regional State. Nurses who worked at selected referral hospitals in Amhara Regional State during the data collection period were included, and nurses who were given free service and not seriously ill during the data collection period were excluded. The sample size was calculated using a single mean population formula $n=\frac{\left(\frac{z a}{2}\right) 2 *(\sigma) 2}{d 2} \quad$ with a consideration of the following assumptions: The level of confidence $(Z \alpha / 2)=95 \%$, and margin of error was 0.07 based on calculation from the previous study $(\sigma=$ standard deviation $=0.65, \mathrm{n}=$ sample size $345 \mathrm{SE}=\sigma / \sqrt{ } \mathrm{n}=0.65 /$ $\sqrt{ } 343=0.035$ and $\sigma^{2}=$ variance of the mean). Therefore, calculated margin of error $(\mathrm{d}) \rightarrow \mathrm{z} \alpha / 2 * \mathrm{SE}=$ which is $0.035 * 1.96=0.068 \approx 0.07$. By taking the overall nurses' cultural competence level mean and standard deviation 
$(1.94 \pm 0.65)$ which was taken from a similar study conducted in Ethiopia. ${ }^{8}$

The final sample size was calculated as follows.

$\mathrm{n}=\frac{\left(\frac{\mathrm{za}}{2}\right) 2 *(\sigma) 2}{\mathrm{~d} 2} \mathrm{n}=(1.96)^{2} *(0.65)^{2} /(0.07)^{2}, \mathrm{n}=3.84 * 0.42 /$ $0.0049=1.61 / 0.0049=n=329$. Using design effect $1.5 \%$ and $10 \%$ non-response rate, the final minimum sample size was $329 * 1.5 * 10 \%$. Therefore, the final sample size of this study was estimated as 543 . The final calculated sample size was proportionally allocated for each selected referral hospitals. The sampling frame was prepared for each hospital by obtaining lists of nurses from the nursing directors and the human resource management office. Finally, the study subjects of (543) selected each hospital using a simple random sampling technique.

For the qualitative data, the purposive sampling technique was used and 10 participants were interviewed.

\section{Data Collection Tool and Procedure}

Data were collected using structured and self-administered English version of the Nurse Cultural Competence Scale Questionnaire (NCCSQ), and cultural care nursing models of Campinha Bacote's Inventory for Assessing the Process of Cultural Competence-Revised (IAPCC-R) were adapted from a related article. ${ }^{18}$ The internal content validity was checked by five nurse experts from different Universities in the country for the previous study. The CCQN consists of a total of 36 items: cultural awareness 7 items, cultural knowledge 10 items, cultural sensitivity 9 items, and cultural skills 10 items. Each item uses a five-point Likert scale to measure participants' responses.

For qualitative data, the interview guide was developed by the principal investigator based on the related literature review. ${ }^{8,19}$ Qualitative data were obtained with open-ended questions with a detailed face-to-face interview by probing questions with the audio recording after obtaining the verbal consent of the participants.

The reliability of the tool was assessed by a pretest, which was conducted on $27(5 \%)$ of the sample size among nurses working in Woldiya Referral Hospital before 1 week of the actual data collection period. The result showed that the Cronbach's alpha of the overall CCQN was 0.91 and each subscale ranged from 0.78 for cultural skill to 0.87 for cultural sensitivity, which was reliable. Data were gathered with a self-administered questionnaire and collected from those experienced in data collection.
For qualitative data, the trustworthiness was obtained with considering; plausible information was drawn from the participants with face-to-face interviews. The findings were transferred to another context with another respondent.

Eight trained (four BSc and four MSc) nurses supervisors' data collectors were employed in the data collection process. The qualitative data were collected by the principal investigator.

\section{Data Processing and Analysis}

The quantitative data were coded and entered to Epi-data manager version 4.6, then exported to SPSS Version 25. The level of overall cultural competence is categorized based on the mean of cultural competence questionnaire for nurses (CCQN) scale score for every four domains and Cultural Competence Assessment Scale, which measure score item by the rank of the highest level achieved; a score of 1 indicates no activity on that criterion, a score of 5 indicates the benchmark standard. Items are added for a final score. ${ }^{20,21}$ The level of each subscale of cultural competence (ie, cultural awareness, cultural knowledge, cultural sensitivity, and cultural skill) was categorized based on the mean of each subscale into four mean scores. ${ }^{20}$ These were the low level cultural competence (mean score $=1-1.80$ ), low to moderate level cultural competence (mean score $=1.81-2.60$ ), moderate level cultural competence (mean score $=2.61-3.40$ ) and high level cultural competence (mean score $>3.41$ ). Data were analyzed using SPSS software with a linear regression model and the descriptive statistics were presented in the form of texts and tables. Independent $t$-test and one-way analysis of variance (ANOVA) were used to examine mean differences between groups of independent variables by checking its assumptions of normality and homogeneity using histogram and values of skewness and kurtosis, and confirmed by Kolmogorov-Smirnov tests and Shapiro-Wilk test. The non-parametric tests like Kruskal-Wallis test and Mann-Whitney $U$-test were used in failed ANOVA and independent $t$-test. The multicollinearity was ensured by the variance inflation factors (VIFs) and ranged from 1.13 to 5.04, which ruled out any issues of multicollinearity. The assumptions of homogeneity were examined by Levene's test of equality of variance, and there were no significantly different variances among groups $(\mathrm{p}=0.064)$. Variables having more than two categories were changed to dummy variables of $\mathrm{k}-1$, where $\mathrm{k}=$ number of 
categories and were entered for multiple regression analysis by assuming the last category as a reference.

Qualitative data were analyzed using open code software for coding and thematic analysis approach. In-depth interviews were held with a semi-structured questionnaire and each interview lasted approximately 25-30 minutes. Their voice was recorded with an audio recorder. The audio recording was transcribed in the local language and translated to English then coded in Open code software. Thematic analyses were preceded after coding data with a codebook in the software as a flexible and helpful full method to obtain a clear and detailed account of qualitative data categories. Similar contents were created and similar ideas were taken to each theme. The findings of the quantitative study were integrated with qualitative findings by triangulation design and were presented congruently.

\section{Result \\ Quantitative Results}

A total of 516 participants were enrolled in the study making the response rate of $95.02 \%$. More than half $(54.5 \% 56.2 \%)$ of the participants were female and aged 20-30 years, respectively. The median age of the participants was 30 years with $\mathrm{IQR}=8.75$. The majority, of the participants, were ethnicity of Amhara (76\%), and Orthodox religion (75.2\%). More than three-fourth (77.3\%) were Bachelor's degrees and one-third $(33.7 \%)$ had work experience in the range of 6-10 years. Most of the participants $(92.1 \%)$ were staff nurses. More than half $(52.7 \%)$ of the participants can speak at least one Ethiopian language other than Amharic and English (Table 1).

The overall cultural competence nursing care of participants was $(\mathrm{M} \pm \mathrm{SD}=3.39 \pm 0.61)$ out of a total possible scores range from 1 to 5 which indicates a moderate level of cultural competency. The level of cultural competence of participants was scored in subscale of cultural awareness $(\mathrm{M} \pm \mathrm{SD}=3.15 \pm 0.87)$, whereas the highest subscale cultural competence was in the area of cultural sensitivity $(\mathrm{M} \pm \mathrm{SD}=3.60 \pm 0.81$ (Table 2 ).

In the multiple linear regressions, variables such as master education level, use of a language other than Amharic and English, frequency of caring diversified patient and feedback system in a hospital were significantly associated with the cultural competency nursing care. In this regard, being a master educational level increases the score of cultural competency care by 0.34 $(\beta=0.34,95 \%$ CI $(0.222-0.462))$. Using a language other than Amharic and English decreased the score of cultural competency care by 0.107 ( $\beta-0.107,95 \%$ CI $(-0.187$, $-0.028)$ ). Having frequency of caring diversified patient decrease the score of cultural competency care by 0.119 $(\beta=-0.119(-0.195,-0.043))$. The presence of feedback systems in the hospital was increased by the score of cultural competency care by $0.195 \quad(\beta=0.195 \quad(0.059$, 0.331)) (Table 3).

Independent $t$-test found that there was a statistically significant difference between mean scores of participants who spoke other than the Amharic and English languages and who could not speak any other Ethiopian language; $(t(514)=1.35, \mathrm{p}<0.02, \mathrm{~d}=0.12)$ (Table 4).

Based on one-way analysis of variance (ANOVA), significant differences were detected for ethnicity, educational level, frequency of giving care for diverse people, and level of health institution served before with a $p$-value $<0.05$. The result of mean comparison with ANOVA revealed that there was a significant difference in mean scores among groups by ethnicity $(F(3,513)=3.13$, $\mathrm{p}=0.025, \mathrm{H \eta}^{2}=0.02$ ) (Table 5).

A Kruskal-Wallis $H$-test (non-parametric for ANOVA) test was done to compare mean differences between variables those were violating normality assumption. Among these variables, only the age of participants was significantly different between age groups $\left(\mathrm{X}^{2}(3)=8.819\right.$, $\mathrm{P}=0.032$ (Table S1).

\section{Qualitative Results}

Ten participants, five male, and five female were involved in this study. They were staff nurses, matrons, head nurses, having experience ranging from 3 to 15 years, and their ages also range from 26 to 40 years (Table 6). Based on coded data, themes were created into four main themes, language barriers, organizational barriers, cultural difference, and health care provider factors. A face-to-face indepth interview was conducted and the voice recordings were arranged, transcribed, and coded after all main themes were identified from the interview (Table S2).

\section{Language Barrier}

Most of the participants mentioned the language barrier as a major problem to give proper nursing care due to lack of communication and no availability of interpreters in the hospital. One participant mentioned that

The major challenge that I have got from culturally and the linguistically different patient is language or 
Table I Socio-Demographic Characteristics of Nurses in Selected Referral Hospitals in Amhara Region, April 202I (n = 5I6)

\begin{tabular}{|c|c|c|c|c|}
\hline Variables & Categories & Median ( \pm IQR) & $\mathbf{N}$ & $\%$ \\
\hline \multirow[t]{3}{*}{ Sex } & Male & & 235 & 45.5 \\
\hline & Female & & 281 & 54.5 \\
\hline & Total & & 516 & 100 \\
\hline \multirow[t]{5}{*}{ Age } & $20-30$ & $30(9)$ & 290 & 56.2 \\
\hline & $31-40$ & & 193 & 37.4 \\
\hline & $4 I-50$ & & 31 & 6 \\
\hline & $\geq 5$ । & & 2 & 0.4 \\
\hline & Total & & 516 & 100 \\
\hline \multirow[t]{5}{*}{ Ethnicity } & Amhara & & 392 & 76.0 \\
\hline & Oromo & & 61 & 11.8 \\
\hline & Tigre & & 30 & 5.8 \\
\hline & Others* & & 33 & 6.4 \\
\hline & Total & & 516 & 100 \\
\hline \multirow[t]{5}{*}{ Religion } & Orthodox & & 388 & 75.2 \\
\hline & Muslim & & 105 & 20.3 \\
\hline & Protestant & & 17 & 3.3 \\
\hline & Others** & & 6 & 1.2 \\
\hline & Total & & 516 & 100 \\
\hline \multirow[t]{5}{*}{ Education level } & Diploma & & 62 & 12.0 \\
\hline & Bachelor's & & 399 & 77.3 \\
\hline & degree & & & \\
\hline & Master's degree & & 55 & 10.7 \\
\hline & Total & & 516 & 100 \\
\hline \multirow[t]{5}{*}{ Work-experience in years } & $<5$ & & 208 & 40.3 \\
\hline & $6-10$ & $6(5)$ & 174 & 33.7 \\
\hline & $11-15$ & & 90 & 17.4 \\
\hline & $>15$ & & 44 & 8.5 \\
\hline & Total & & 516 & 100 \\
\hline \multirow[t]{5}{*}{ Current role } & Matron nurse & & 3 & 0.6 \\
\hline & Supervisor nurse & & 12 & 2.3 \\
\hline & Head nurse & & 26 & 5.0 \\
\hline & Staff nurse & & 475 & 92.1 \\
\hline & Total & & 516 & 100 \\
\hline \multirow[t]{3}{*}{ Ability to speak any language(s) other than Amharic and English } & Yes & & 272 & 52.7 \\
\hline & No & & 244 & 47.3 \\
\hline & Total & & 516 & 100 \\
\hline \multirow[t]{5}{*}{ Types of a spoken language other than Amharic and English ( $n=272)$} & Oromifa & & 112 & 41.2 \\
\hline & Tigrigna & & 51 & 18.8 \\
\hline & Agewugna & & 62 & 22.7 \\
\hline & Other*** & & 47 & 17.3 \\
\hline & Total & & 272 & 100 \\
\hline \multirow[t]{3}{*}{ Ever learned about cultural care nursing } & Yes & & 213 & 41.3 \\
\hline & No & & 303 & 58.7 \\
\hline & Total & & 516 & 100 \\
\hline \multirow[t]{3}{*}{ Experience with caring for patients with different ethnicity and culture } & Yes & & 354 & 68.6 \\
\hline & No & & 162 & 31.4 \\
\hline & Total & & 516 & 100 \\
\hline
\end{tabular}

(Continued) 
Table I (Continued).

\begin{tabular}{|c|c|c|c|c|}
\hline Variables & Categories & Median ( \pm IQR) & $\mathbf{N}$ & $\%$ \\
\hline \multirow[t]{9}{*}{ Frequency of caring for diverse patients $(n=354)$} & Almost & & 96 & 27.1 \\
\hline & every day & & & \\
\hline & I to 2 times & & 115 & 32.5 \\
\hline & a week & & & \\
\hline & I to 2 times & & 89 & 25.1 \\
\hline & a month & & & \\
\hline & Several times & & 54 & 15.3 \\
\hline & a year & & & \\
\hline & Total & & 354 & 100 \\
\hline \multirow[t]{3}{*}{ Working other than current hospital } & Yes & & 361 & 70.0 \\
\hline & No & & 155 & 30.0 \\
\hline & Total & & 516 & 100 \\
\hline \multirow[t]{6}{*}{ Level of health institution working before $(n=361)$} & Tertiary hospital & & 46 & 12.8 \\
\hline & General hospital & & 77 & 21.4 \\
\hline & Primary hospital & & 175 & 48.6 \\
\hline & Health center & & 62 & 17.2 \\
\hline & and other & & & \\
\hline & Total & & 360 & 100 \\
\hline \multirow[t]{3}{*}{ Cultural care-related in-service training } & Yes & & 142 & 27.5 \\
\hline & No & & 374 & 72.5 \\
\hline & Total & & 516 & \\
\hline \multirow[t]{3}{*}{ Use of translator while giving nursing care } & Yes & & 274 & 53.1 \\
\hline & No & & 242 & 46.9 \\
\hline & Total & & 516 & \\
\hline \multirow[t]{6}{*}{ Type of translator used while giving care $(n=274)$} & Family & & 162 & 31.4 \\
\hline & Colleagues & & 70 & 13.6 \\
\hline & Medical & & 27 & 5.2 \\
\hline & translator & & & \\
\hline & Others**** & & 15 & 2.9 \\
\hline & Total & & 274 & 100 \\
\hline \multirow[t]{3}{*}{ Culture care plan in your hospital } & Yes & & 224 & 43.4 \\
\hline & No & & 292 & 56.6 \\
\hline & Total & & 516 & \\
\hline \multirow[t]{3}{*}{ Presence and use of culture-based guidelines in hospital $(n=224)$} & Yes & & 80 & 35.7 \\
\hline & No & & 144 & 64.3 \\
\hline & Total & & 224 & 100 \\
\hline \multirow[t]{3}{*}{ Presence of feedback system in a hospital } & Yes & & 121 & 23.4 \\
\hline & No & & 395 & 76.6 \\
\hline & Total & & 516 & 100 \\
\hline
\end{tabular}

Notes: *Himtegn, Gumz, Agew; **Catholic, Wake feta; ***Himtegna, Ge'ez and Arabic; ****Nursing students, attendants of other patients and any individual available and volunteer.

communication barrier and they want to practice their own culture, and then I try to solve this challenges with getting translator from their family, colleagues, and others. I waste more time to solve such problem. (P 4)

Another participant supports this idea as

even if the patient can speak Amharic they have different dialects and sayings which are stranger for health professionals which is making difficult the communication even cannot be interpreted. When we see that the language and speech tone, there is a difference by which sometimes in our hospital patients use high pitch tone of voice while talking and a stranger for this area may consider it as the patient is becoming angry "laughing". (P5).

The presence of different dialects is also one barrier even if patients can talk Amharic some words are difficult to 
Table 2 Descriptive Statistics for Each Subscale and the Total Scores of the CCQN Scale $(n=516)$

\begin{tabular}{|l|l|l|l|l|l|}
\hline Variables & Possible Range & Minimum & Maximum & Mean (SD) & Level of Cultural Competence \\
\hline Cultural awareness & $1-5$ & 1.00 & 4.86 & $3.15(0.87)$ & Moderate level \\
Cultural knowledge & $1-5$ & 1.00 & 4.90 & $3.4 I(0.73)$ & Within high level \\
Cultural skill & $1-5$ & 1.22 & 5.00 & $3.40(0.73)$ & Moderate level \\
Cultural sensitivity & $1-5$ & 1.00 & 5.00 & $3.60(0.8 I)$ & Highest level \\
Total CCQN scale score & $1-5$ & 1.00 & 4.90 & $3.39(0.6 \mathrm{I})$ & Moderate level \\
\hline
\end{tabular}

Table 3 Factors Associated with Cultural Competency in Multiple Regression Models

\begin{tabular}{|l|l|l|l|l|l|l|l|}
\hline Independent Variables & \multicolumn{2}{l|}{$\begin{array}{l}\text { Unstandardized } \\
\text { Coefficients }\end{array}$} & $\begin{array}{l}\text { Standardized } \\
\text { Coefficients }\end{array}$ & $\boldsymbol{t}$ & \multicolumn{2}{l|}{$\mathbf{9 5 \%}$ CI } \\
\cline { 2 - 5 } & $\mathbf{B}$ & Std. Error & Beta & & & LB & UB \\
\cline { 2 - 7 } Education (bachelor's degree) & 0.169 & 0.043 & 0.127 & 3.918 & 0.000 & 0.085 & 0.254 \\
Education (master's degree) & 0.342 & 0.061 & 0.195 & 5.596 & 0.000 & 0.222 & 0.462 \\
Language other than Amharic and English & -0.107 & 0.040 & -0.096 & -2.654 & 0.008 & -0.187 & -0.028 \\
Frequency of caring, diversified patient & -0.119 & 0.039 & -0.079 & -3.059 & 0.002 & -0.195 & -0.043 \\
Culture care plan & -0.101 & 0.059 & -0.082 & -1.712 & 0.087 & -0.216 & 0.015 \\
Feedback system in a hospital & 0.195 & 0.069 & 0.135 & 2.812 & 0.005 & 0.059 & 0.331 \\
\hline
\end{tabular}

Abbreviations: LB, lower bound; UB, upper bound.

Table 4 Independent Sample t-test: Mean Differences of Grouped Variables Predicting Cultural Competence of Nurses Working in Selected Referral Hospitals in Amhara Region, Ethiopia, 202I ( $n=516)$

\begin{tabular}{|c|c|c|c|c|c|c|c|c|}
\hline Variables & Categories & $\mathbf{N}$ & $M(S D)$ & $\boldsymbol{t}$ & $d f$ & $\mathbf{p}$ & d & $95 \% \mathrm{Cl}$ \\
\hline \multirow[t]{3}{*}{ Languages are spoken other than Amharic and English } & Yes & 271 & $\begin{array}{l}3.43 \\
\pm .64\end{array}$ & - & - & - & - & - \\
\hline & No & 245 & $\begin{array}{l}3.36 \\
\pm .57\end{array}$ & - & - & - & - & - \\
\hline & Total & 516 & $\begin{array}{l}3.39 \\
\pm .61\end{array}$ & 1.35 & 514 & 0.02 & 0.12 & $\begin{array}{l}-0.033- \\
0.178\end{array}$ \\
\hline \multirow[t]{3}{*}{$\begin{array}{l}\text { Experience of caring for culturally and ethically different from } \\
\text { yours }\end{array}$} & Yes & 354 & $\begin{array}{l}3.44 \\
\pm .58\end{array}$ & - & - & - & - & - \\
\hline & No & 162 & $\begin{array}{l}3.29 \\
\pm .65\end{array}$ & - & - & - & - & - \\
\hline & Total & 516 & $\begin{array}{l}3.39 \\
\pm .61\end{array}$ & 2.58 & 514 & 0.01 & 0.24 & $0.06-0.43$ \\
\hline \multirow[t]{3}{*}{ Use of language translator } & Yes & 274 & $3.47 \pm 57$ & - & - & - & - & - \\
\hline & No & 242 & $3.31 \pm 65$ & - & - & - & - & - \\
\hline & Total & 516 & $3.39 \pm 61$ & 2.86 & 514 & 0.004 & 0.25 & $0.08-0.42$ \\
\hline \multirow[t]{3}{*}{ Availability of feedback system in a hospital } & Yes & 121 & $\begin{array}{l}3.28 \\
\pm .69\end{array}$ & - & - & - & - & - \\
\hline & No & 395 & $\begin{array}{l}3.42 \\
\pm .65\end{array}$ & - & - & - & - & - \\
\hline & Total & 516 & $3.39 \pm 61$ & -2.32 & 514 & 0.02 & 0.24 & $\begin{array}{l}-0.27 I- \\
0.022\end{array}$ \\
\hline \multirow[t]{3}{*}{ Presence of culture care plan } & Yes & 224 & $3.45 \pm 56$ & - & - & - & - & - \\
\hline & No & 292 & $3.34 \pm 64$ & - & - & - & - & - \\
\hline & Total & 516 & $3.39 \pm 61$ & 2.06 & 514 & 0.04 & 0.2 & $0.008-0.356$ \\
\hline
\end{tabular}

Abbreviations: M, mean; SD, standard deviation; N, sample size; $t$, $t$-distribution; $p$ value, alpha (level of significance); df, degree of freedom for distribution table; $d$, Cohen's $\mathrm{d}$ (effect size of two group variable); $\mathrm{Cl}$, confidence interval of $95 \%$. 
Table 5 Analysis of Variance (ANOVA): Factors Associated with Cultural Competence of Nurses Working in Referral Hospitals in Amhara Region, Ethiopia, $2021(n=516)$

\begin{tabular}{|c|c|c|c|c|c|c|c|c|c|c|}
\hline Variables & Categories & $\mathbf{N}$ & (M士SD) & Groups & SS & $d f$ & MS & $\boldsymbol{F}$ & $\boldsymbol{P}$ & $\eta^{2}$ \\
\hline \multirow[t]{4}{*}{ Education level } & Diploma & 62 & $3.17 \pm .67$ & Between groups & 7.32 & 2 & 3.66 & 10.12 & 0.001 & 0.038 \\
\hline & Bachelor's degree & 399 & $3.39 \pm .60$ & & & & & & & \\
\hline & Masters' degree & 55 & $3.67 \pm .51$ & Within a group & 185.579 & & 0.362 & & & \\
\hline & Total & 516 & & & & & & & & \\
\hline \multirow[t]{4}{*}{ Level of health institution } & Tertiary hospital & 46 & $3.27 \pm .90$ & Between groups & 3.722 & 3 & 1.24 & 3.23 & 0.02 & 0.027 \\
\hline & General hospital & 77 & $3.30 \pm 64$ & & & & & & & \\
\hline & Primary hospital & 175 & $3.44 \pm .54$ & Within groups & 136.433 & & 0.38 & & 0.03 & 0.04 \\
\hline & Health center & 62 & $3.58 \pm .51$ & & & & & & & \\
\hline
\end{tabular}

Abbreviations: M, mean; SD, standard deviation; SS, sum of square; $d f$, degree of freedom; MS, mean of square; $F$ test, alpha level; $\eta^{2}$, eta square for effect size.

Table 6 Socio-Demographic Characteristics of Nurses Working in Referral Hospital in Amhara Region, Ethiopia, 202I ( $n=10$ )

\begin{tabular}{|l|l|l|l|l|l|}
\hline Participants' Code & Gender & Age & Level of Education & Work Experence & Current Role \\
\hline I - I & F & 28 & BSc & 6 & Staff \\
I - & F & 40 & BSc & I5 & Head \\
I - 3 & M & 35 & BSc & II & Matron \\
I - 4 & M & 29 & BSc & 9 & Vice matron \\
I - 5 & M & 33 & BSc & II & Head \\
I - 6 & M & 35 & BSc & 8 & Staff \\
I - & M & 32 & MSc & 4 & Staff \\
I - 8 & F & F & MSc & 3 & Staff \\
I - 9 & F & 26 & BSc & II & Staff \\
\hline
\end{tabular}

interpret and sometimes they used uncommon terms, this was stated by one percipient. "I have an experienced patient come from other ethnic group and they have a dialect to call males as female and vice versa, when it happens some nurses those do not have experience will be laughing and the patient becomes worried" (p 3). Among all cultural issues language was the most difficult barrier during giving nursing care. One participant mentions this

When I give care for those patients who come from a different area with a different language I try to get a person who can speak patient's language, for example, their friends or my colleagues. There is no interpretation service in our hospital and there is no training for us about culture-based care. (P 10)

\section{Organizational Barriers}

The standard for cultural competence in a health care setting is intended to be inclusive for all cultural aspects beyond the medical treatment, such as languages, beliefs, and values. ${ }^{22}$ In this study, hospitals were not considered culture-based care starting from the absence of language interpreters and nursing care plans considering patients' culture. Some of the respondents were mentioned that there were structured ways to support culture-based care in their working hospital. One participant mentions this,

While I give nursing care in a daily manner there is a challenge that patients have different cultural and religious interests in some situations I permit some religious activities such as praying but the hospital setup is not comfortable for such practice. Sometimes patients are against medical treatment instead they prefer to go to the home and try to traditional options. (P 6)

This idea was supported by another participant as explained as

Some patients need care based on their cultural way and we can try to do this as possible as we do but some time there are barriers to implement such practices like hospital set up. (P 4)

Another participant stated that "Unavailability of the interpreter is the most challenging issue in our hospital" (P 8). 


\section{Cultural Difference}

Culture influences not only health practices but also how the healthcare provider and the patient perceive illness. Additionally, becoming aware of own attitudes and tendencies to the stereotype about different cultural groups allows providing genuine care and concern to give culturally competent care. Nurses should be aware of intercultural nursing care. Participants state this idea as

First of all when I try to give the care I try to see their demographic data to identify their religion, culture, and language. Next, I ask them what they do before coming to the hospital and what traditions they follow. To know their language, firstly I greet them and evaluate the fluent speech, if not I try to get translators, but there is no formal interpreter in our hospital. If there is prolonged duration, I try to speak key words of patient's language. (P 1)

Another participant supports this idea as

I consider patients will have different in their culture since our hospital serves patients come from different areas including across the border of the country like Sudan so that we consider language and try to communicate in English other ways we use a translator. (P 2).

To identify and solve problems related to religion and culture-based differences, stated by one key informant as follows:

As a religious difference exists we cannot discuss openly, but I have an experience of some professionals make a bias for their religion, "hmmmmm" in general this is the difficult idea to mention. (P 5)

Another key informant said that

cultural related difference is a reason for delayed to give appropriate care: Some patients believe that traditional healers are more perfect than medical care and refuse our treatment but we try to convince to have planned treatment. Sometimes we permit patients to refuse and send home but they come back with complications. Some patients also believe that the disease results with evil spirit and they prefer spiritual care and we permit the treatment concomitantly with medical care. (P 4)

\section{Health Care Providers-Related Factor}

Nurses' educational level, training obtained, being able to speak a different language, and having empathy to the patient were important to have cultural competence health care services to produce the quality of care, but sometimes health care providers ignore these useful characteristics intentionally or unknowingly. In this study, participants state that there is no training for cultural care, but nationwide compassionate care is declared in all health care facility, but negligence is observed. One participant states that

I always serve my patient honestly and fulfill their interest. When I give care to those who are coming from other areas and have different cultures, beliefs, and religions, there is a gap to fulfill their need such as the interest of going religious but the hospital did not prepare it and some professionals neglect this interest. (P 6)

Another respondent said that

Training is one way of capacity building for health care professionals, it might be in-service training or in the formal education. Even if all health care providers are trained for compassionate care in our hospital there is negligence deliberately or unknowingly. (P 7)

This idea was supported by another participant "some professionals having experience with giving care for diverse patient expected to give better care than other. Due to the existence of cultural differences like language differences sometimes nurses are faced with difficulty to give care with a patient's language (p10).

\section{Discussion}

The results of this study showed that participants were at a moderate level of cultural competence in nursing care. The lowest score of cultural competency subscale was cultural awareness, whereas the highest score subscale was cultural sensitivity. This finding was in line with a study conducted in the USA. ${ }^{23}$ The possible explanation because that respecting the uniqueness of the patient and treating them with a closed approach rather than selfexamination of their cultural background can improve cultural competency in nursing care.

In the multiple linear regressions, variables such as master educational level, use of a language other than Amharic and English, and feedback system in a hospital were significantly associated with the cultural competency nursing care. In this regard, being a master educational level increases the score of cultural competency care by 0.34 other levels of education. This finding is supported by the study done in China. ${ }^{24}$ The possible explanation might be because nurses with a high level of education might have a chance to obtain school training to give culturally competent care. Using a language other than Amharic and English decreased the score of cultural competency care 
by 0.107 . This finding was supported by the study conducted in South Africa that reported that nurses from nonEnglish-speaking backgrounds scored significantly higher in cultural competence nursing care than English-speaking nurses. ${ }^{25}$ This might be because nurses who speak the local language in addition to the national language give better care for their patients. The qualitative findings of this study support that the language barrier was the main factor to give care to diverse patients. This finding was in line with a study conducted in Saudi Arabia that showed that critical care nurses experienced difficulty in communicating due to language differences. ${ }^{26}$ The qualitative finding also showed how patients feel while their language is not properly used. Regarding this one key informant states that "patients come from the different area having their language and dialects talks by their way, someone will laugh and they become angry" which leads to unsatisfied with the care provided. This finding is supported by the study conducted in $\mathrm{China}^{24}$ which states that disrespecting patients' language and inappropriate use of terms can lead to stereotyping and misunderstandings. This study finding is also supported by other related studies conducted in Iran and Germany, ${ }^{27,28}$ which states that the presence of multi-linguistic patients coming from different areas from the country and out of the country could affect cultural competency nursing care. In this study, participants explained that referral hospitals were served to patients coming from different areas within the region and out of the region with different cultures, values, and language backgrounds, which make difficult to give culturally congruent care due to the absence of an interpreter, originated from lack of interpreter and training related with a culture-based care for nurses. This finding was supported by a qualitative study conducted in Taiwan. ${ }^{29}$ This might be because nurses do not understand how patients can place their beliefs above themselves, and standard clinical procedures can result in becoming conflicted. The presence of feedback systems in the hospital was increased by the score of cultural competency care by 0.195. This finding was supported by a report of a systematic review conducted across Europe. ${ }^{30}$ This might be because feedback as part of a multifaceted intervention may improve compliance with desired practice, in comparison with no intervention.

Nurses who worked in the primary health care system increased by an effect size of 0.027 than others other work settings $\left(\mathrm{H \eta}^{2}=0.027\right)$. This finding was similar to the study done in Thailand. ${ }^{20}$ The possible explanation might be because of having an opportunity to listen and learn the way of life and culture of the patients who live in a specific area. Participants with the age of 20-30 years (mean rank 244.4, $\mathrm{p}=0.03$,) had more culturally competent nursing care than other age groups. This finding was in contrast with the studies done in Thailand and China, ${ }^{20,24}$ in which nurses who were working for more than 10 years were more in cultural nursing care. This discrepancy could be due to prolonged exposure to diverse patients and might get advanced training than in our setups and prolonged service makes nurses burnout.

The finding in this study confirmed that there was a perfect correlation between cultural sensitivity and cultural competence and a moderate correlation with cultural knowledge $(r=1, r=0.45, p<0.001)$ respectively. This finding was inconsistent with the study done in the USA, which states that correlation between cultural knowledge and competence of provider. ${ }^{31}$ This discrepancy could be due to curriculum differences, availability of in-service training, and the presence of practice guidelines. Cultural sensitivity was the highest subscale score in this study, which was similar to the study done in Thailand. ${ }^{20}$ The possible reason might be due to sharing of local cultures, which means nurses who worked in primary care in a different area shared some values, religion, and culture in the community helps to give culturally competent care in the health care setting.

The qualitative result also explores lack of training is a barrier for giving culturally competent care even if nurses are trained for professional ethics and compassionate care, and values related to religion affect the care given by nurses because some patients refuse some activities in the ward such as "taking medications before the meal is forbidden in the fasting day", which was supported by a similar study conducted in Turkey. ${ }^{32}$ The possible reason might be because the nurses had more problems in areas related to language barriers, health perception about the disease, and religious beliefs when providing health care services. The interview finding in this study also showed that health facilities related problem stated by one key informant nurse "patients who cannot be communicated needs an interpreter but not available in the hospital". These findings were supported by research done in the USA. ${ }^{33}$ This might be because health care employers often lack resources to provide culturally and linguistically appropriate services, which might be not having enough interpreters for the diverse patient and inadequate 
understanding of health literacy and cultural norms essential for the populations served.

\section{Limitation of the Study}

Since cultural competent care is a continuous and ongoing practice, it is difficult to assess at the point of time of data collection, therefore this cross-sectional study design is not enough to elaborate the observed gap rather a longitudinal observational study with a checklist might be a better study design.

\section{Conclusion}

The overall cultural competence was at a moderate level, among four subscales cultural sensitivity was the highest. Able to speak any Ethiopian language other than Amharic and English, advanced education level, using interpreter, following the culture care plan, working in another health facility before the current hospital, and presence of feedback system were major factors for cultural competence nursing care. The presence of different cultures, language and lack of organizational supports were the major identified barriers to cultural competence nursing care.

Nurses who worked in primary health care were more culturally competent than others. Patients came from the same area but had different beliefs; culture, language, and use of their dialect were major barriers to culturally competent care. The policymakers ought to incorporate cultural care plan, facilitation of language interpreters, and receiving feedback from the customer about the service will ensure cultural competency nursing care.

\section{Implication for Nursing Practice}

Cultural competency nursing care is important by fostering mutual respect, understanding, and trust; promoting inclusion of patient and family responsibility for their health; and increasing community participation and involvement in health issues. The finding of this study showed that nurses' education level, use of language interpreters, and feedback system were a significant association with cultural competency nursing care. In addition, language barriers, absence of nursing care plans considering patient's culture, lack of honesty, and empathy were the barriers of cultural competency in nursing care. Therefore, updating the nursing educational level, use of nursing interpreter during the patient assessment, formulating and integrating nursing care plans considering patient's culture and being honest and empathetic for patients who had cultural differences have their implications on health policy, to change the current practice and better quality patient care.

\section{Abbreviations}

ANOVA, analysis of variance; CCC-PRE-R, Clinical Cultural Competency Questionnaire - Revised; CCQN, Cultural Competency Questionnaire for a Nurse; CLAS, culturally and linguistically appropriate services; CSA, Central Statistical Agency; CSRH, compressive specialized hospital; IAPCCR-R, Inventory for Assessing the Process of Cultural Competence-revised; NCCSQ, Nurse Cultural Competence Scale Questionnaire; PTTM, Papadopoulos Tilki and Taylor's model; RH, referral hospital; SPSS, Statistical Package for Social Science; UoGCSH, University of Gondar Comprehensive Specialized Hospital.

\section{Data Sharing Statement}

The raw data are available from the corresponding author on reasonable request, the summary of data is presented in the manuscript.

\section{Ethical Approval and Consent}

The study was performed based on the ethical standards put down under the declaration of Helsinki. The ethical clearance was obtained from the school of nursing research and ethical review committee on behalf of the University of Gondar research and ethical review committee with reference No-S/N 164/7/2013. Official permission letters were obtained from each selected referral hospital. After understanding the purpose of the study, a signed written informed consent was obtained from the study participants, which ensured that no identities were known by the investigators, and any response of the participants was anonymized. Confidentiality of participants' information was kept.

\section{Acknowledgments}

Our deepest gratitude goes to University of Gondar for all expenses of this research work and to the study participants who participated in the research. The authors also would like to give great appreciation to data collectors and supervisors for their contributions to this paper.

\section{Author Contributions}

All authors made a significant contribution to the work reported, whether that is in the conception, study design, execution, acquisition of data, analysis and interpretation, or in all these areas; took part in drafting, revising or 
critically reviewing the article; gave final approval of the version to be published; have agreed on the journal to which the article has been submitted; and agree to be accountable for all aspects of the work.

\section{Funding}

The study was supported by the University of Gondar College of Medicine and Health Sciences. The funder did not have a further role in the design, data collection, analysis, drafting, manuscript, preparation, or publication of this paper.

\section{Disclosure}

The authors report no conflicts of interest for this work.

\section{References}

1. Campinha-Bacote J. A culturally conscious model of mentoring. Nurse Educ. 2010;35(3):130-135. doi:10.1097/NNE.0b013e3181d950bf

2. Ngo-Metzger Q, Telfair J, Sorkin DH, et al. Cultural competency and quality of care: obtaining the patient's perspective. Commonw Fund. 2006;963:1-50.

3. Cai D-Y. A concept analysis of cultural competence. Int J Nurs Sci. 2016;3(3):268-273. doi:10.1016/j.ijnss.2016.08.002

4. Zazzi E. Contributors to the development of intercultural competence in nursing students. Nurse Educ Today. 2020;90:104424. doi:10.1016/ j.nedt.2020.104424

5. Nickitas DM, Pontes NM. Around the corner, across the globe: developing global citizens through civic engagement. Metrop Univ. 2020;31(2):53-70. doi:10.18060/23813

6. Jongen C, McCalman J, Bainbridge R. Health workforce cultural competency interventions: a systematic scoping review. $B M C$ Health Serv Res. 2018;18(1):1-15. doi:10.1186/s12913-018-3001-5

7. Pitriani U, Ibrahim K, Pebrianti S. Cultural competency and nursing care among Sundanese nurses ethnic group in Indonesia. Malahayati Int $j$ Nurs Health Sci. 2020;3(2):131-137. doi:10.33024/minh. v3i2.3634

8. Aragaw A, Yigzaw T, Tetemke D. Cultural competence among maternal healthcare providers in Bahir Dar city administration, Northwest Ethiopia: a cross-sectional study. BMC Pregnancy Childbirth. 2015;15(1):1-10. doi:10.1186/s12884-015-0643-8

9. Makary MA, Daniel M. Medical error-the third leading cause of death in the US. BMJ. 2016;353:i2139.

10. Shen Z. Cultural competence models and cultural competence assessment instruments in nursing: a literature review. $J$ Transcult Nurs. 2015;26(3):308-321. doi:10.1177/1043659614524790

11. Seright T. Perspectives of registered nurse cultural competence in a rural state-Part II. Online J Rural Nurs Health Care. 2012;7 (1):57-69. doi:10.14574/ojrnhc.v7i1.144

12. Eborall H, Wobi F, Ellis K, et al. Integrated screening of migrants for multiple infectious diseases: qualitative study of a city-wide program. EClin Med. 2020;21:100315. doi:10.1016/j.eclinm.2020.100315

13. Henderson S, Horne M, Hills R, Kendall E. Cultural competence in healthcare in the community: a concept analysis. Health Soc Care Community. 2018;26(4):590-603. doi:10.1111/hsc.12556

14. PragashnieGovender DM. Exploring cultural competence amongst OT students. Occup Ther Int. 2017;1(2179781):8.
15. Chen H-C, McAdams-Jones D, Tay DL, Packer JM. The impact of service-learning on students' cultural competence. Teach Learn Nurs. 2012;7(2):67-73. doi:10.1016/j.teln.2011.11.002

16. Shepherd SM, Willis-Esqueda C, Newton D, Sivasubramaniam D, Paradies Y. The challenge of cultural competence in the workplace: perspectives of healthcare providers. BMC Health Serv Res. 2019;19 (1):1-11. doi:10.1186/s12913-019-3959-7

17. Kaihlanen A-M, Hietapakka L, Heponiemi T. Increasing cultural awareness: a qualitative study of nurses' perceptions about cultural competence training. BMC Nurs. 2019;18(1):1-9. doi:10.1186/ s12912-019-0363-x

18. Papadopoulo T. Transcultural Care: A Guide for Health Care Professionals. Quay Books; 1998:0515.

19. Torsvik M, Hedlund M. Cultural encounters in reflective dialogue about nursing care: a qualitative study. $J$ Adv Nurs. 2008;63 (4):389-396. doi:10.1111/j.1365-2648.2008.04723.x

20. Songwathana P, Siriphan S. Thai nurses' cultural competency in caring for clients living in a multicultural setting. Pac Rim Int J Nurs Res. 2015;19(1):19-31.

21. Haugland G, Institute NK. Cultural competence assessment tools. center of excellence for cultural competence; 2010.

22. Polacek GN, Martinez R. Assessing cultural competence at a local hospital system in the United States. Health Care Manag. 2009;28 (2):98-110. doi:10.1097/HCM.0b013e3181a2cb32

23. Heitzler ET. Cultural competence of obstetric and neonatal nurses. J Obstet Gynecol Neonatal Nurs. 2017;46(3):423-433. doi:10.1016/j. jogn.2016.11.015

24. Cai D, He W, Klug D. Cultural competence among nurses and its influencing factors: a cross-sectional study. Nurs Health Sci. 2021;23:411-418. doi:10.1111/nhs. 12821

25. De Beer J, Chipps J. A survey of cultural competence of critical care nurses in KwaZulu-Natal. South Afr J Crit Care. 2014;30(2):50-54. doi:10.7196/SAJCC.188

26. Albougami AS, Pounds K, Alotaibi J. Comparison of four cultural competence models in transcultural nursing: a discussion paper. Int Arch Nurs Health Care. 2016;2(3):1-5. doi:10.23937/2469$5823 / 1510053$

27. Amiri R, Heydari A, Dehghan-Nayeri N, Vedadhir AA, Kareshki H. Challenges of transcultural caring among health workers in Mashhad-Iran: a qualitative study. Glob J Health Sci. 2016;8 (7):203. doi:10.5539/gjhs.v8n7p203

28. Mews C, Schuster S, Vajda C, et al. Cultural competence and global health: perspectives for medical education-Position paper of the GMA committee on cultural competence and global health. GMS J Med Educ. 2018;35(3). doi:10.3205/zma001174

29. Lin M-H, Wu C-Y, Hsu H-C. Exploring the experiences of cultural competence among clinical nurses in Taiwan. Appl Nurs Res. 2019;45:6-11. doi:10.1016/j.apnr.2018.11.001

30. Flottorp SA, Jamtvedt G, Gibis B, McKee M. Using audit and feedback to health professionals to improve the quality and safety of health care. World Health Organization; 2010.

31. Castro A, Ruiz E. The effects of nurse practitioner cultural competence on Latina patient satisfaction. J Am Acad Nurse Pract. 2009;21 (5):278-286. doi:10.1111/j.1745-7599.2009.00406.x

32. Yilmaz M, Toksoy S, Direk ZD, Bezirgan S, Boylu M. Cultural sensitivity among clinical nurses: a descriptive study. $J$ Nurs Scholarsh. 2017;49(2):153-161. doi:10.1111/jnu.12276

33. Young S, Guo KL. Cultural diversity training: the necessity of cultural competence for health care providers and in nursing practice. Health Care Manag. 2020;39(2):100-108. doi:10.1097/ HCM.0000000000000294 


\section{Publish your work in this journal}

Nursing: Research and Reviews is an international, peer-reviewed, open access journal publishing original research, reports, reviews and commentaries on all aspects of nursing and patient care. These include patient education and counseling, ethics, management and organizational issues, diagnostics and prescribing, health outcomes, economics and resource management, improving patient safety in all settings. The manuscript management system is completely online and includes a very quick and fair peer-review system. Visit http://www. dovepress.com/testimonials.php to read real quotes from published authors.

Submit your manuscript here: http://www.dovepress.com/nursing-research-and-reviews-journal 